\title{
Analysis of local ionospheric time varying characteristics with singular value decomposition
}

\author{
Jakob Jakobsen · Per Knudsen • Anna B. O. Jensen
}

Received: 8 January 2009 / Accepted: 10 March 2010 / Published online: 23 April 2010

(c) The Author(s) 2010. This article is published with open access at Springerlink.com

\begin{abstract}
In this paper, a time series from 1999 to 2007 of absolute total electron content (TEC) values has been computed and analyzed using singular value decomposition (SVD). The data set has been computed using a Kalman Filter and is based on dual frequency GPS data from three reference stations in Denmark located in the midlatitude region. The station separation between the three stations is $132-208 \mathrm{~km}$ (the time series of the TEC can be freely downloaded at http:// www.heisesgade.dk). For each year, a SVD has been performed on the TEC time series in order to identify the three time varying (daily, yearly, and 11 yearly) characteristics of the ionosphere. The applied SVD analysis provides a new method for separating the daily from the yearly components. The first singular value is very dominant (approximately six times larger than the second singular value), and this singular value corresponds clearly to the variation of the daily cycle over the year. The second singular value corresponds to variations of the width of the daily peak over the year, and the third singular value shows a clear yearly variation of the daily signal with peaks around the equinoxes. The singular values for each year show a very strong correlation with the
\end{abstract}

Electronic supplementary material The online version of this article (doi:10.1007/s00190-010-0378-2) contains supplementary material, which is available to authorized users.

J. Jakobsen (凶) • P. Knudsen

National Space Institute, Technical University of Denmark,

Copenhagen, Denmark

e-mail: jj@space.dtu.dk

URL: http://www.heisesgade.dk

P. Knudsen

e-mail: pk@space.dtu.dk

\section{A. B. O. Jensen}

AJ Geomatics, Brønshøj, Denmark

e-mail: aj@ajgeomatics.com

URL: http://www.ajgeomatics.com sunspot number for all the singular values. The correlation coefficients for the first 5 sets of singular values are all above 0.96. Based on the SVD analysis yearly models of the TEC in the ionosphere can be recomposed and illustrate the three time varying characteristics of the ionosphere very clearly. By prediction of the yearly mean sunspot number, future yearly models can also be predicted. These can serve as a priori information for a real time space weather service providing information of the current status of the ionosphere. They will improve the Kalman filter processing making it more robust, but can also be used as starting values in the initialization phase in case of gaps in the data stream. Furthermore, the models can be used to detect variations from the normal local ionospheric activity.

Keywords GNSS - Time varying ionosphere - Kalman filter $\cdot$ Singular value decomposition

\section{Introduction}

GNSS satellite signals are affected while being transmitted through the ionosphere, the part of the Earth's atmosphere extending from the surface of the Earth to an altitude of about $1,000 \mathrm{~km}$. The effect is a function of the amount of free electrons along the signal path which is measured by the total electron content (TEC). See for instance Hunsucker and Hargreaves (2003) for an introduction to the ionosphere.

The ionosphere is dispersive for radio waves, and for the GPS signals the ionospheric effect is therefore different for the L1 and L2 frequencies. The main effects on the satellite signals are the group delay and the phase advance. These are equal in size, but with opposite sign for the code and phase observations. An introduction to the ionospheric effect on GNSS signals is provided for instance by Misra and 
Enge (2006). Further, satellite signals can also be affected by small scale irregularities in the ionosphere, which can cause severe amplitude fading and phase scintillation effects. See for instance Yeh and Liu (1982) or Aarons (1982) for an introduction and global morphology of the phenomena.

The ionospheric effect on the satellite signals induces an error which must be handled in the positioning process when GNSS is used for positioning and navigation. With data from permanent continuously operating GNSS reference stations, where the station coordinates are very well estimated, the ionospheric effect on the satellite signals can be extracted and used for ionospheric modelling. Several techniques for estimation of TEC from GNSS satellites signals have been published, see for instance Komjathy (1997) or Schaer (1999).

For grid based regional or global ionosphere modelling various interpolation techniques are used, for instance linear interpolation or multiquadric interpolation as described by Skone (1998). Other examples are statistically based spatial interpolation techniques like kriging, which can be used for the generation of a global ionospheric model (Orus et al. 2005). The use of spherical harmonics for global or large scale models is also well known and discussed, e.g. by Schaer (1999).

Precise ionospheric modeling will improve the accuracy of the position of single-frequency GNSS receivers (Lou 2001). Furthermore precise ionospheric modeling is also important for other space-based observation systems as well as for communication systems and space weather studies. The observed rapid evolution of the ionosphere currently defies sufficient modeling to allow its prediction to future time epochs and therefore studies of its time varying behavior are needed.

The ionosphere, in the midlatitude region, is in its nature varying over time. The main characteristics include the daily cycle with a peak at 14:00 local time and a low, almost constant activity, during nighttime. Further, a yearly cycle with peaks around equinoxes, and finally a 11-year time period correlated with the sunspot number as described in e.g. (Hargreaves 1992).

The speed of the propagation of radio waves depends upon the number of free electrons. The TEC is defined as the number of electrons in a tube of $1 \mathrm{~m}^{2}$ cross section extending from the receiver $u$ to the satellite $s$, see Eq. (1) from Kaplan and Hegarty (2006)

$\mathrm{TEC}=\int_{u}^{s} n_{\mathrm{e}}(l) \mathrm{d} l$

where $n_{\mathrm{e}}(l)$ is the electron density along the signal path. TEC is measured in TEC units (TECU). 1 TECU equals $10^{16}$ electrons $/ \mathrm{m}^{2}$.

The ionospheric delay, $\delta_{\mathrm{I}}$ expressed in measurements of carrier phase can be written (to a first order) as in Eq. (2).
$\delta_{\mathrm{I}}=-\frac{40.3 \cdot \mathrm{TEC}}{f^{2}}$

where $f$ is the frequency of the signal. See for instance Hofmann-Wellenhof et al. (2008).

The TEC has different characteristics for varying latitude and thus an important factor for obtaining a local accurate model is to use data from local GNSS reference stations, as described in Misra and Enge (2006). For instance, in Denmark at approximately $56^{\circ} \mathrm{N}$ latitude the length of daylight varies considerably during the year, compared to lower latitudes. Therefore, the daily cycle of the ionosphere varies more over the year. Also, in the case of a real time space weather service, providing information of the local current status of the ionosphere, it is important to have a good local a priori knowledge of the time varying characteristics of the ionosphere. This knowledge can be used to make the data processing in a Kalman filter more robust and to estimate parameters in cases where the filter needs to be reinitialized. The a priori information can be generated by looking at local time series of the time varying ionosphere.

\section{Data and location}

Observations from three dual frequency GPS receivers have been used. The reference stations are located in Denmark with a station separation of approximately $132-208 \mathrm{~km}$. The triangle and geographic relation can be seen in Fig. 1. The GPS observations are stored in the RINEX format with a sampling rate of $30 \mathrm{~s}$, and an elevation mask of $15^{\circ}$ has been

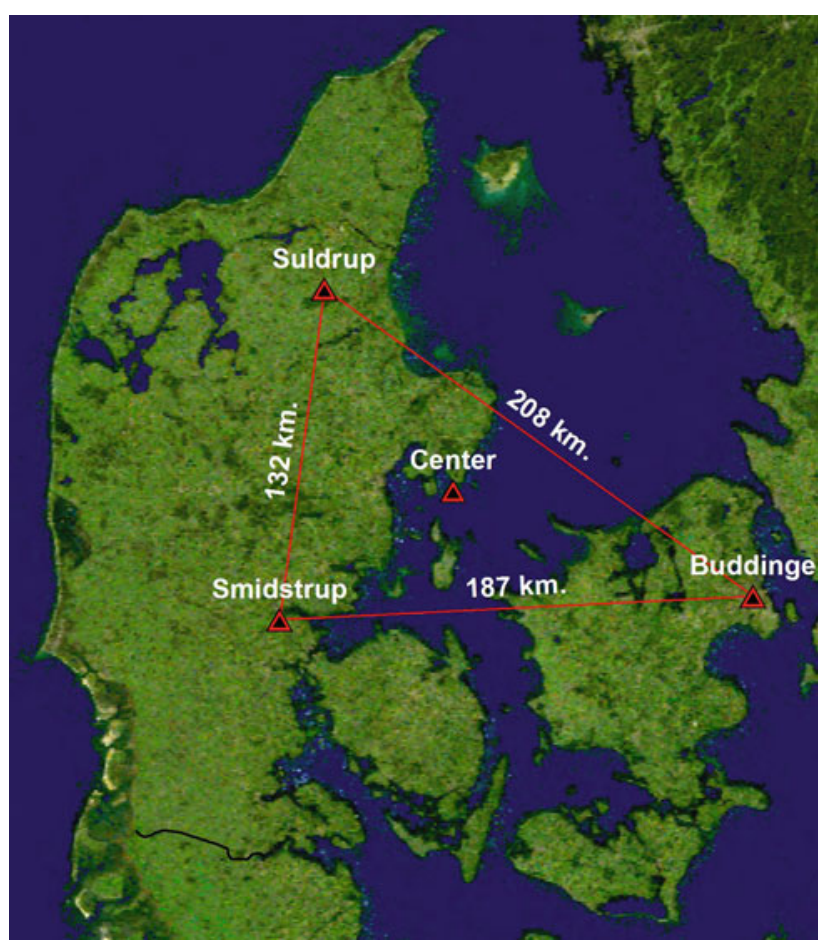

Fig. 1 Reference stations in Denmark used in the data processing 
applied. Three stations have been used, instead of just one, in order to provide a better estimate of the ionosphere above the center mark. The stations are maintained and operated by the National Survey and Cadastre in Denmark.

The center mark of the triangle is at approximately $56^{\circ} \mathrm{N}$ latitude, just below the auroral oval under normal ionospheric activity. During storm periods, the auroral oval can extend to lower latitudes and affect the GNSS signals received in Denmark. During these periods the time varying characteristics of the ionosphere will be different.

\section{Computation of the time series}

A time series of TEC values is computed using a Kalman filter. The principle for the observation model, simplified to include one receiver, is shown in Fig. 2. A single layer model, where the ionosphere is compressed into an infinitesimal shell at the peak of the electron density, is used. In this data processing, the peak is assumed to be at a height of $400 \mathrm{~km}$. The ionospheric pierce point, IPP, is the intersection of the shell and a direct line from the satellite (SV) to the GNSS receiver (U1).

The observation equations for the two carrier phase measurements, $L 1$ and $L 2$, can be expressed in units of meters for satellite $s$ and receiver $u$ as, see Feng (2008).

$$
\begin{aligned}
L 1_{u}^{s}= & \rho_{u}^{s}+\delta_{\mathrm{orb}, u}^{s}+c\left(\delta t^{s}-\delta t_{u}\right)+\delta_{\mathrm{I}, u, 1}^{s}+\delta_{\text {trop }, u, 1} \\
& +\lambda_{1}\left(N_{u, 1}^{s}+\varphi_{u, 1}-\varphi_{1}^{s}\right)+\epsilon_{u, 1} \\
L 2_{u}^{s}= & \rho_{u}^{s}+\delta_{\mathrm{orb}, u}^{s}+c\left(\delta t^{s}-\delta t_{u}\right)+\delta_{\mathrm{I}, u, 2}^{s}+\delta_{\text {trop }, u, 2} \\
& +\lambda_{2}\left(N_{u, 2}^{s}+\varphi_{u, 2}-\varphi_{2}^{s}\right)+\epsilon_{u, 2}
\end{aligned}
$$

where $\rho$ is the geometric distance, $\delta_{\text {orb }}$ is the orbital error, $c$ is the speed of radio waves in vacuum, $\delta t$ is the clock error

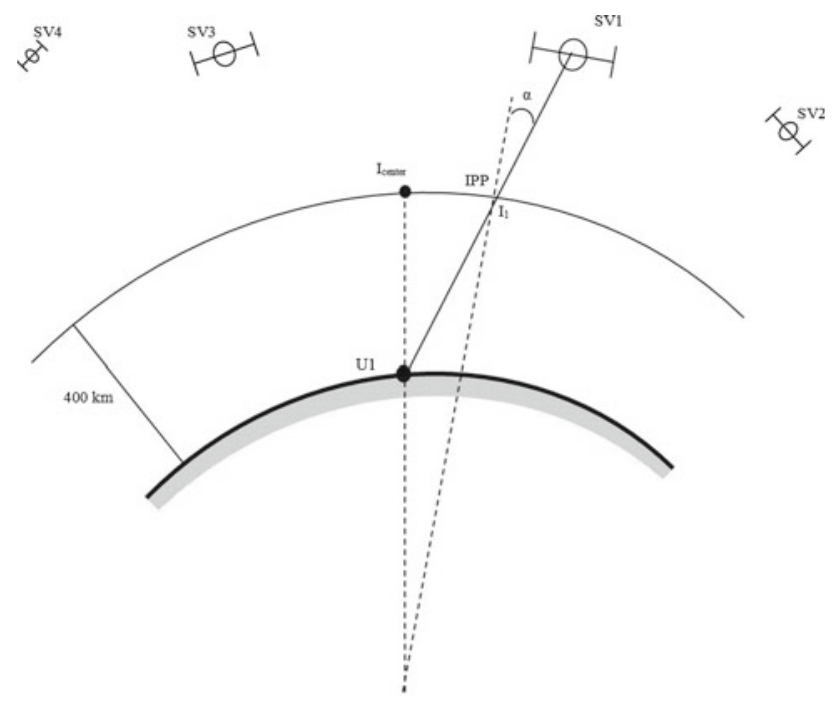

Fig. 2 Illustration of the observation model for one receiver (Seeber 2003) for all components, $\delta_{\mathrm{I}}$ is the ionospheric phase delay, $\delta_{\text {trop }}$ is the tropospheric propagation delay, $\lambda$ is the wavelength, $N$ is the integer ambiguity, $\varphi$ is the initial phase, $\epsilon$ is the receiver phase noise, including the phase multipath, subscript ${ }_{1}$ and 2 refer to the $L 1$ and $L 2$ carriers.

By taking the difference of Eqs. (3) and (4), the geometry free solution $L 4$ can then be expressed as in Eqs. (5)-(7), see Figs. 1 and 2 and the Eqs. (2), (3), and (4).

$$
\begin{aligned}
L 4_{u}^{s}= & L 1_{u}^{s}-L 2_{u}^{s} \\
= & 40.3\left(\frac{1}{f_{2}^{2}}-\frac{1}{f_{1}^{2}}\right) \mathrm{TEC} \\
& +\lambda_{1}\left(N_{1}^{s}+\phi_{1, u}-\phi_{1}^{s}\right)-\lambda_{2}\left(N_{2}^{s}+\phi_{2, u}-\phi_{2}^{s}\right) \\
= & c n v \cdot \operatorname{map}\left(\alpha_{u}^{s}\right)\left(\mathrm{TEC}_{\mathrm{cen}, \mathrm{vert}}+\frac{\partial \mathrm{TEC}}{\partial \mathrm{lat}}\left(\mathrm{lat}_{u}^{s}-\mathrm{lat}_{\mathrm{cen}}\right)\right. \\
& \left.+\frac{\partial \mathrm{TEC}}{\partial \operatorname{lon}}\left(\operatorname{lon}_{u}^{s}-\operatorname{lon}_{\mathrm{cen}}\right)\right)+c_{u}^{s}
\end{aligned}
$$

where $c n v$ is given by $40.3\left(\frac{1}{f_{2}^{2}}-\frac{1}{f_{1}^{2}}\right)$, map is the mapping function given by $\frac{1}{\cos \alpha}$ (see Seeber 2003), $\alpha$ is the zenith angle at the IPP, subscript cen refers to the center of the triangle, subscript vert refers to the vertical value, lat and lon are the latitude and longitude, $c$ is a constant.

The $L 4$ observation consists mainly of the delay caused by the ionosphere. In Eq. (7), this delay is split into three parts in order to represent the TEC at the center point. These are the absolute TEC and the gradients in the latitude and longitude of the TEC. The connection between the slant delays and the vertical is performed by the mapping function.

Another part of the $L 4$ observation is the constant $c$. This constant consists of the initial phase for the satellites and receivers and the integer ambiguities. It is assumed that the initial phases do not change from one epoch to another and that no cycle slips occur. It is therefore only the three ionospheric parts, that vary from epoch to epoch.

The number of observations for $m$ epochs, each with $n$ visible satellites will be $n \cdot m$, and the number of unknowns will be $n+3 m$. The unknowns can therefore over time be estimated using a Kalman filter. The Kalman filter is a dynamic filter that essentially can be described by five equations given in Eqs. (8)-(12) (see Welch and Bishop 2006).

$$
\begin{aligned}
& \mathbf{K}_{k}=\frac{\mathbf{P}_{k}^{-} \mathbf{H}_{k}^{T}}{\mathbf{H}_{k} \mathbf{P}_{k}^{-} \mathbf{H}_{k}^{T}+\mathbf{R}_{k}} \\
& \hat{\mathbf{x}}_{k}=\hat{\mathbf{x}}_{k}^{-}+\mathbf{K}_{k}\left(\mathbf{z}_{k}-\mathbf{H}_{k} \hat{\mathbf{x}}_{k}^{-}\right) \\
& \mathbf{P}_{k}=\left[\mathbf{I}-\mathbf{K}_{k} \mathbf{H}_{k}\right] \mathbf{P}_{k}^{-} \\
& \hat{\mathbf{x}}_{k+1}^{-}=\mathbf{A}_{k} \hat{\mathbf{x}}_{k}+\mathbf{B}_{k} u_{k} \\
& \mathbf{P}_{k+1}^{-}=\mathbf{A}_{k} \mathbf{P}_{k} \mathbf{A}^{T}+\mathbf{Q}_{k}
\end{aligned}
$$

In this processing the term $\mathbf{B}_{k} u_{k}$ in Eq. (11) is set to zero, because there is no input from observations outside 
the system. The A matrix equals the identity matrix, because it is assumed that there will be no change in the ionosphere from epoch to epoch. The connection between the observations, $z_{k}$, and the estimates, $\hat{x}_{k}$, the $\mathbf{H}$ matrix, is provided in Eqs. (13) and (14). The observations are given in Eq. (7).

$$
\begin{aligned}
\mathbf{H} & =\left[\begin{array}{ccc}
c n v \cdot \operatorname{map}\left(\alpha_{u}^{s 1}\right) & \ldots & \\
c n v \cdot \operatorname{map}\left(\alpha_{u}^{s 1}\right)\left(\operatorname{lat}_{u}^{s 1}-\operatorname{lat}_{\mathrm{cen}}\right) & \ldots & \\
c n v \cdot \operatorname{map}\left(\alpha_{u}^{s 1}\right)\left(\operatorname{lon}_{u}^{s 1}-\operatorname{lon}_{\mathrm{cen}}\right) & \ldots & \\
1 & 0 & \ldots \\
0 & 1 & \ldots \\
\vdots & \vdots & \ddots
\end{array}\right] \\
\hat{x} & =\left[\begin{array}{c}
\mathrm{TEC}_{\mathrm{cen}, \mathrm{vert}} \\
\frac{\partial \mathrm{TEC}}{\text { Ilat }} \\
\frac{\partial \mathrm{TEC}}{\partial \operatorname{long}} \\
c_{u}^{s 1} \\
c_{u}^{s 2} \\
\vdots
\end{array}\right]
\end{aligned}
$$

For an explanation of the remaining variables the reader is referred to Welch and Bishop (2006).

The observation model was implemented in the Kalman filter using Matlab, and nine full years of data from the GNSS reference stations, described above, were processed to obtain absolute vertical TEC values for the center point every $30 \mathrm{~s}$ through the 9 years. When a cycle slip was detected the observation from the relevant satellite was reinitialized for future epochs. The parameters for the Kalman filter were saved for every processed hour and reused when a new RINEX file was loaded.

During the processing of the three time series, data gaps in the RINEX files were observed. Values originating from initialization of the Kalman filter immediately after the data gaps, have been removed. The computed TEC time series from the entire period 1999-2007 can be freely downloaded from http://www.heisesgade.dk.

The processed time series of vertical TEC has been verified by comparing it to the final global ionospheric maps produced by the International GNSS Service, IGS (see http://www.igscb.jpl.nasa.gov/components/prods.html for further explanation). The maps are available every second hour, and the vertical TEC has been extracted at the location of the considered center point for all of 2003. The difference between the two time series has a standard deviation of 1.9 TECU and is normally distributed with an offset of 1.8 TECU. The correlation coefficient between the two time series is 0.96 . The IGS maps are global and the long wavelength of the ionospheric variation is weighted higher. An offset to a momentary value from a local model must therefore be expected. This verification of the processed TEC time series is considered satisfactory in order to perform the singular value decomposition analysis.

\section{SVD analysis on the TEC time series}

The singular value decomposition (SVD) is a technique for decomposing a matrix into a product of three factors. A plot of the elements will reveal recognizable patterns, when the data set corresponds to samples of continuous variables. This is described by, e.g. Preisendorfer and Mobley (1988).

For a given $m \times n, m \geq n$ real valued matrix A the equation for SVD of $\mathbf{A}$ can be written as in Eq. (15) from Parker (2004).

$\mathbf{A}=\mathbf{U S V}^{\mathrm{T}}$

where $\mathbf{U}$ is a $m \times m$ matrix, $\mathbf{V}$ is a $n \times n$ matrix, $\mathbf{S}$ is a diagonal matrix of size $m \times n$. $\mathbf{U}$ and $\mathbf{V}$ are both orthogonal matrices. $\mathbf{S}$ is a diagonal matrix of nonnegative values, called the singular values of $\mathbf{A}$, and all other values are zero.

The TEC time series, computed using the Kalman filter, has been organized into 9 yearly $96 \times 365$ matrices, where each of the 96 values per day are the mean over $15 \mathrm{~min}$. Each yearly matrix corresponds to the A matrix in Eq. (15). The $\mathbf{U}$ matrix will correspond to the daily variation and the $\mathbf{V}$ matrix will correspond to the yearly variation. The SVD is performed using the LAPACK routine (Anderson et al. 1999).

\section{Results}

An example of the computed TEC time series can be seen in Fig. 3. Three summer days as well as three winter days for 2001, 2004, and 2007 are illustrated. The 24 hour daily signal, the width of the daily peak between summer and winter time, and the size of the daily signal for high, medium, and low ionospheric activity is clearly visible.

In Fig. 4, the result for the SVD analysis is illustrated. Three years (2001, 2004, and 2007) have been chosen in order to represent high, medium, and low ionospheric activity. For the results for all years, the reader is referred to the additional information (electronic supplementary material). The first singular value is illustrated on the left side. A very clear daily signal of the total electron content is observed in the $\mathbf{U}$ matrix. This is expected as the Kalman filter smoothes the data sequentially, epoch by epoch. The signal has approximately the same shape and magnitude for all the 9years, which also can be inferred from the figure. It should be noted, that the signal is always positive, meaning that this signal will always be present.

In the $\mathbf{V}$ matrix for the first singular value, it is observed how this daily signal varies through the year. The signal is noisy indicating that there is a very low correlation of the size of the daily signal from day to day. The signal can be divided into two trends based on the years with high or with low ionospheric activity. From 1999 to 2003 (represented by year 2001 in the figure), the signal is higher during the 
Fig. 3 Example of the computed time series in UTC time for three summer days (Day of year 173-175) and three winter days (Day of year 11-13) in 2001,2004 , and 2007

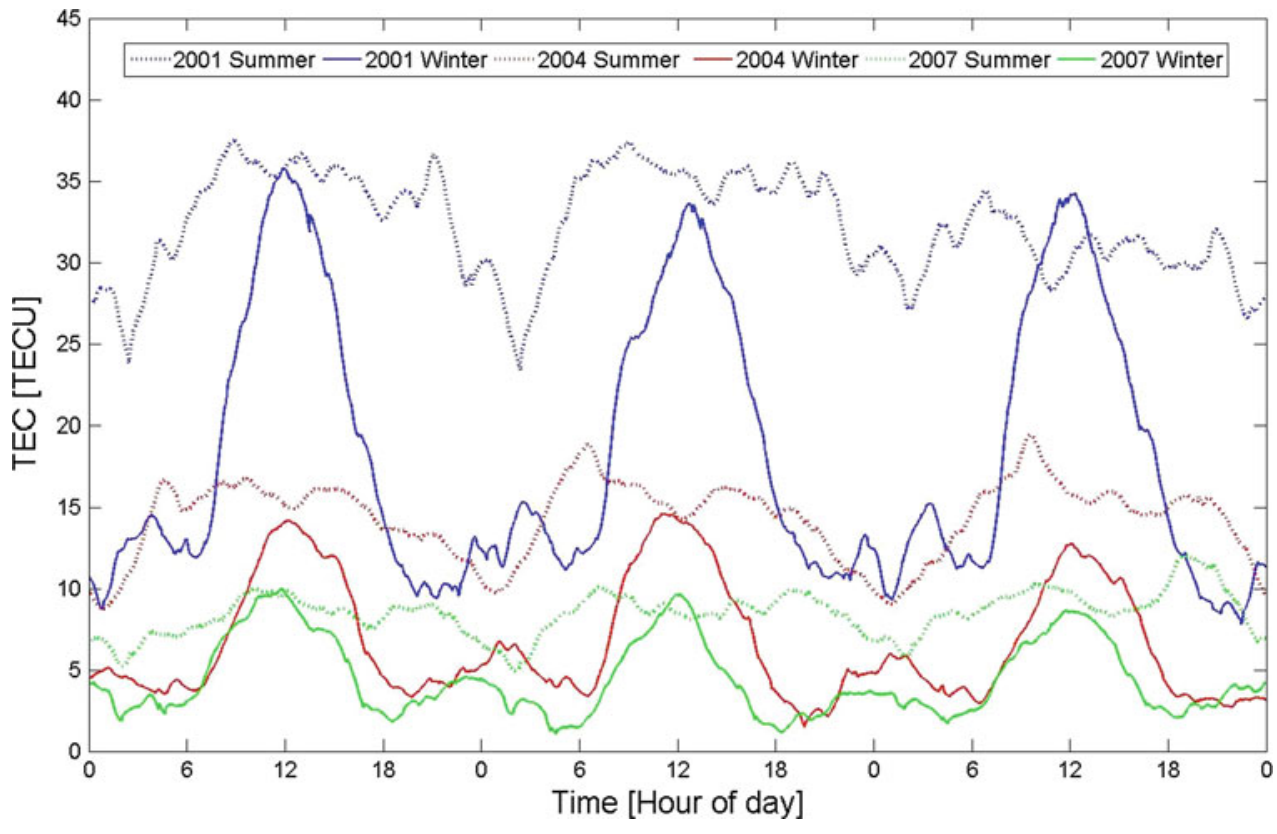

$\mathrm{U}, \mathrm{V}, \mathrm{S}$ for the first three singular values for three selected years
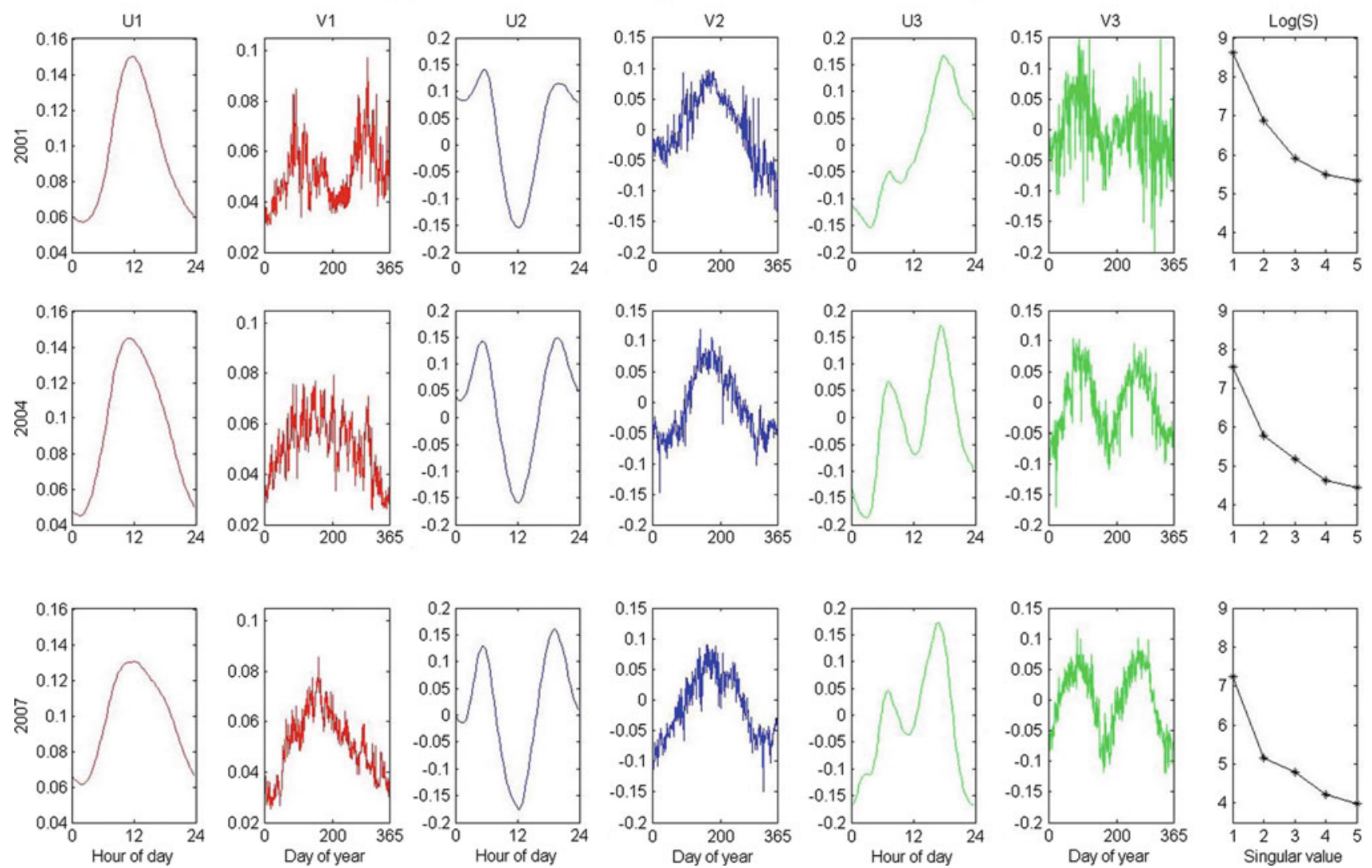

Fig. $4 \mathbf{U}$ and $\mathbf{V}$ matrices for the first three singular values for three selected years. The time axis is in UTC time for the $\mathbf{U}$ matrices and in day of year for the $\mathbf{V}$ matrices. The first five singular values in the $\mathbf{S}$ matrix are illustrated to the right

equinoxes and from 2004 to 2007 is the daily signal stronger in the early summer. Furthermore, the signal is less noisy for 2007 than for 2004. This can be interpreted as during solar max the influence from the solar wind is the dominating factor. This influence is semiannual as described by Rishbeth et al. (2000). During more relaxed solar activity 
Table 1 Correlation coefficients for the singular values for each year and the yearly mean sunspot number

\begin{tabular}{llllll}
\hline Singular value & 1 & 2 & 3 & 4 & 5 \\
\hline Corr. Coef. & 0.96 & 0.97 & 0.98 & 0.99 & 0.96
\end{tabular}

years, the UV radiation becomes more dominant indicated by the reduced noise for 2004 and 2007 and the signal becomes annual.

The first five singular values are illustrated to the right on Fig. 4. The ratio between the first and the second is approximately six meaning, that the first singular value dominates the time series for the total electron content. The singular values for each year also show another behavior. Each set of singular values $1-5$ over the 9 -year period has been correlated with the yearly mean sunspot number. The correlation coefficients for the first five singular values are listed in Table 1, but the remaining singular values (up to 96) show similar behavior. The correlation coefficients indicate a strong correlation of the total electron content with the sunspot number. ${ }^{1}$

For the second singular value, see Fig. 4 in the middle, another clear daily signal is observed in the $\mathbf{U}$ matrix over the years. The signal is positive in the morning and in the evening and negative during midday. Thus, this signal will determine the width of the daily peak as well as it will damp the peak at 1400 hours local time. The yearly signal in the $\mathbf{V}$ matrix also shows a clear trend over the years. It is highest in the summer and lowest in the winter. The signal has the same low noise for all three years and the day to day correlation is therefore better. This corresponds very well to the variation caused by the length of daylight in the midlatitude region, which is the same for all the years and varies over the year very smoothly.

The third singular value is shown to the right in Fig. 4. The daily signal in the $\mathbf{U}$ matrix, shows two maxima. The second maximum is very clear for all the years while the first maximum is similar for the years 2004 and 2007. The signal is both positive and negative, meaning that it will correct the daily signal as shown for the first singular value. It will correct the afternoon depletion of the electron content, so the depletion will appear slower. When looking at the yearly trend, in the $\mathbf{V}$ matrix, it can be observed that the depletion will be emphasized during the equinoxes. The yearly signal also shows less noise for the years with less ionospheric activity.

For the fourth and following singular values, there are no recognizable patterns over the years in the daily and the yearly signals. They can therefore be considered as noise and will be discarded in the recomposition of the ionospheric models.

1 The used Sunspot Number is the monthly mean number from the Space Weather Prediction Center (http://www.swpc.noaa.gov/ftpdir/ weekly/RecentIndices.txt).

\section{Recomposition of yearly ionospheric models}

Based on the SVD analysis, where the shape and daily variation over the year was identified for the first three singular values, a model of the time varying ionosphere for each year can be recomposed. The resulting local TEC models for three selected years are illustrated in Fig. 5. The daily signal over the year has been smoothed in order to obtain a yearly trend and to suppress the day to day variation of the size of the daily signal. For an illustration of all the years, the reader is referred to the additional information (electronic supplementary material).

The models clearly illustrate the three ionospheric time varying characteristics. Especially, not only the effect from the 11-year period can be seen with significant higher values for 2001, but also the effects during the equinoxes are more clear during 2001. It can also be verified that the variation caused by the length of daylight is similar for all of the models. The relative effect from the second singular value,
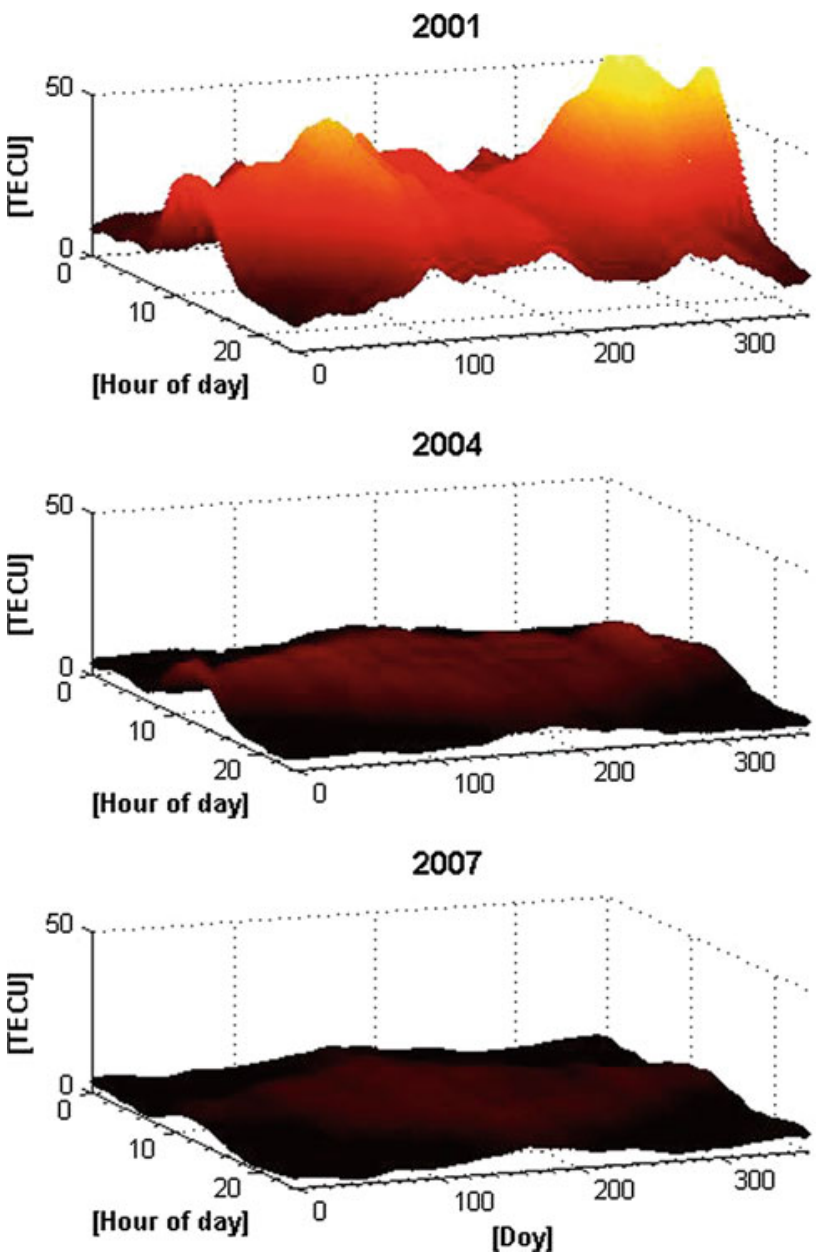

Fig. 5 Recomposed ionospheric models for three selected years based on the first three singular values 
Fig. 6 Example of two events in 2001. The first row shows the residual signal, the second shows the differentiation of the residual signal with respect to time, the third shows the geomagnetic observation data, and the fourth shows the recomposed model

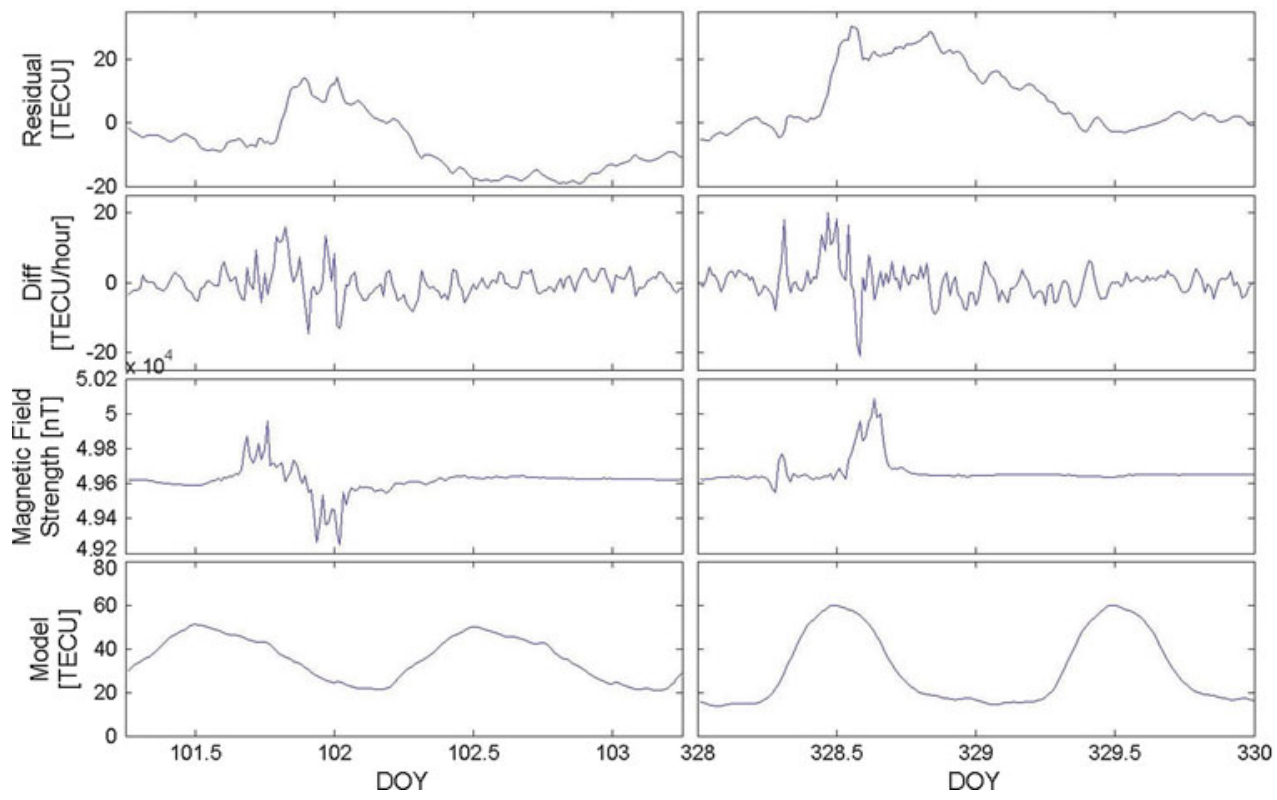

the damping of the 1400 hour local peak, is more visible for 2004 and 2007, than it is for 2001.

An important factor for the time varying characteristics of the ionosphere is the day to day variation. The recomposed models can be used to detect variations from the normal activity, which is of interest for analysis of local space weather studies as well as space weather predictions.

Subtracting the recomposed models from the original time series results in a residual matrix. In Fig. 6, two events during 2001 are illustrated. The first is the evening/nigth between 11th and 12th of April and the second is in the midday/afternoon the 24th of November. Both events are major storms with high $K_{p}$ values and an impact of -270 and $-220 \mathrm{nT}$ in the Dst index. ${ }^{2}$ The residual signal, the differentiation of the residual signal with respect to time, the geomagnetic observations, ${ }^{3}$ and the recomposed model is illustrated for both events.

For the April event, an increase in the TEC from normal activity is observed in the evening, which decreases during the night and becomes negative until the middle of the next day. The differentiated residual signal shows, that the TECU values are fluctuating more in the evening, which are correlated with the local geomagnetic observations. The same correlation between fluctuating TEC and geomagnetic observations are observed for the November event.

These two events also show correlation with global indexes, but this is not consistent for all studied events during 2001. This indicates, that local storms in Denmark can be

\footnotetext{
2 The indices used are from the World Data Center for geomagnetism, Kyoto, http://wdc.kugi.kyoto-u.ac.jp/.

${ }^{3}$ Data are downloaded from the World Data Center for Geomagnetism, Edinburgh, for the observatory Brorfelde at latitude 55.625 and longitude 11.672.
}

detected and using a combination of local TEC observations with local geomagnetic observations will give a more reliable estimation of the ionosphere variation, than when using global indices or models.

\section{Conclusion and future work}

The SVD analysis performed on the computed TEC time series provides a new method for separating the daily from the yearly components. The first singular value is very dominant (approximately six times larger than the second singular value) and this singular value corresponds clearly to how the daily cycle varies over the year. The second singular value corresponds to how the width of the daily peak varies over the year and the third singular value shows a clear yearly variation of the daily signal with peaks around the equinoxes.

The singular values for each year show a very strong correlation with the mean yearly sunspot number with correlation coefficients above 0.96 for the first five sets of singular values.

These three signals, together with the mean sunspot number, can be used to recompose local yearly models of the TEC. In this work, models have been recomposed for Denmark, but the same analysis should be applied at other latitudes and regions in order to validate the procedure and models.

By using a prediction of the yearly mean sunspot number future yearly models can be predicted. This can be used for a real time space weather service providing information of the current local status of the ionosphere. It will contribute to a more robust processing of the Kalman filter by providing information of the general change from one epoch to the next and can be used to generate starting values during initialization of the Kalman filter. 
The models can also be used to detect abnormal variations in the ionosphere by looking at the difference between the model and the observed value, the residual. A correlation between the differentiated residual and local external geomagnetic observations has been found for a number of events during 2001. This shows the potential for combining these two different types of local observations to provide a more reliable local space weather service.

Acknowledgments C. C. Tscherning, University of Copenhagen, is acknowledged for his contribution in the SVD analysis and for his comments through the work. P. Jarlemark from the Technical Research Institute of Sweden is acknowledged for giving access to his expert knowledge of the Kalman filter. The remote Sensing and Geomagnetism group at the Danish Meteorological Institute is acknowledged for making the geomagnetic observations available.

Open Access This article is distributed under the terms of the Creative Commons Attribution Noncommercial License which permits any noncommercial use, distribution, and reproduction in any medium, provided the original author(s) and source are credited.

\section{References}

Aarons J (1982) Global morphology of ionospheric scintillations. Proc IEEE 70(4):360-378

Anderson E, Bai Z, Bischof C, Blackford S, Demmel J, Dongarra J, Croz JD, Greenbaum A, Hammarling S, A McKenney DS (1999) LAPACK users guide, vol 3. Society for Industrial and Applied Mathematics, Philadelphia

Feng Y (2008) Gnss three carrier ambiguity resolution using ionosphere-reduced virtual signals. J Geodesy 82:847-862

Hargreaves J (1992) Principles of ionosphere. Cambridge University Press, Cambridge

Hofmann-Wellenhof B, Lichtenegger H, Wasle E (2008) GNSSglobal navigation satellite systems. Springer, Wien
Hunsucker R, Hargreaves J (2003) The high-latitude ionosphere and its effects on radio propagation. Cambridge University Press, Cambridge

Kaplan E, Hegarty C (2006) Understanding GPS, principles and applications. 2. Artech House, INC, Norwood

Komjathy A (1997) Global ionosphere total electron content mapping using the global positioning system. Technical Report no. 188, Department of Geodesy and Geomatics Engineering, University of New Brunswick

Lou N (2001) Precise relative positioning of multiple moving platforms using gps carrier phase observables. Tech. Rep. 20147, University of Calgary

Misra P, Enge P (2006) Global positioning system, signals, measurements and performance, 2nd edn. Ganga-Jamuna Press, Lincoln

Orus R, Hernandez-Pajarees M, Juan JN, Sanz J (2005) Improvement of global ionospheric vtec maps by using kriging interpolation technique. J Atmos Solar Terr Phys 67:1598-1609

Parker RL (2004) Geophysical inverse theory. Princeton University Press, Princeton

Preisendorfer R, Mobley CD (1988) Principal component analysis in meteorology and oceanography. Elsevier, Amsterdam

Rishbeth H, Muller-Wodarg CF, Zou L, Fuller-Rowell TJ, Millward GH, Moffett RJ, Idenden DW, Aylward AD (2000) Annual and semiannual variations in the ionospheric F2 layer: II. physical discussion. Ann Geophys 18:945-956

Schaer S (1999) Mapping and predicting the earth's ionosphere using the global positioning system. Ph.D. Thesis, Astronomical Institute of the University of Berne

Seeber G (2003) Satellite geodesy, vol 2. Walter de Gruyter, GmbH, Berlin

Skone S (1998) Wide area ionosphere grid modelling in the auroral region. UCGE Reports, Number 20123, Department of Geomatics Engineering, The University of Calgary

Welch G, Bishop G (2006) An introduction to the Kalman filter. Department of Computer Science, University of North Carolina

Yeh KC, Liu C (1982) Radio wave scintillations in the ionosphere. Proc IEEE 70(4):324-360 